\title{
Polycystic ovarian syndrome and insulin resistance: a South Indian study
}

\author{
V. Anuradha ${ }^{1}, \operatorname{Keshav}_{\text {Gangadharan }}{ }^{1 *}$, Rajiv Kumar Saxena ${ }^{2}$, L. Krishna $^{1}$
}

\author{
${ }^{1}$ Department of Obstetrics and Gynecology, PES Institute of Medical Sciences and Research, Kuppam, Andhra \\ Pradesh, India \\ ${ }^{2}$ Department of Obstetrics and Gynecology, MVJ Institute of Medical Sciences, Hoskote, Bangalore, Karnataka, India
}

Received: 19 February 2020

Accepted: 29 February 2020

*Correspondence:

Dr. Keshav Gangadharan,

E-mail: drkeshav@gmail.com

Copyright: (C) the author(s), publisher and licensee Medip Academy. This is an open-access article distributed under the terms of the Creative Commons Attribution Non-Commercial License, which permits unrestricted non-commercial use, distribution, and reproduction in any medium, provided the original work is properly cited.

\begin{abstract}
Background: Polycystic ovary syndrome (PCOS) is a common endocrine disorder in women of reproductive age. Some studies have characterized different aspects of women presenting with PCOS. In this study we characterise the association of insulin resistance (IR) in patients with PCOS in the southern Indian state of Andhra Pradesh.

Methods: A total of 50 women diagnosed to have PCOS according to Rotterdam criteria were studied. IR was estimated using Homeostatic model assessment - insulin resistance (HOMA-IR) and clinical characteristics were recorded.

Results: The prevalence of IR among the study population was $36 \%$. All PCOS patients with IR were overweight or obese, and had impaired glycaemic status, $75 \%$ of PCOS patients with IR also had features of hirsutism.

Conclusions: Considering the prevalence of IR, obesity and impaired fasting glucose in women with PCOS, early institution of treatment by lifestyle changes or medication would lead to improvement in reproductive and metabolic abnormalities.
\end{abstract}

Keywords: Hirsutism, Homeostatic model assessment - insulin resistance, Insulin resistance, Polycystic ovary syndrome

\section{INTRODUCTION}

Polycystic ovarian syndrome (PCOS) is a multifaceted disorder found among women of the reproductive age. It is predominantly a lifestyle disorder and highly prevalent among middle and high income urban and rural population. $^{1}$

Since the report by Burghen et al, that PCOS was associated with hyperinsulinemia, this syndrome has been associated with major metabolic and reproductive comorbidities. Hyperinsulinemia and insulin resistance (IR), commonly associated with PCOS, are responsible for early onset of type 2 diabetes mellitus (DM), dyslipidaemia, cardiovascular disease, osteoporosis and infertility. ${ }^{2,3}$

Even though IR seems to be a central figure in the etiology of PCOS, the triggering factor and the chronology of events which lead to PCOS still remain shrouded in mystery. Sedentary lifestyle and obesity have often been blamed for this malady. Clinical observations support the hypothesis that there is a genetic component to PCOS and the IR associated with it. The polycystic ovarian morphology is suspected to be inherited as an autosomal dominant trait. ${ }^{4}$ The presence of hyperinsulinemia in PCOS women, independent of obesity, has been reported by many studies, suggesting 
that hyperinsulinemia has an impact on ovarian morphology as well as on ovarian function. This may also be responsible for altering $\mathrm{P} 450 \mathrm{c} 17$ activity that results in hyperandrogenism. However, at least $50 \%$ of PCOS women do not have this defect in insulin action. 5,6

The objective of this study was to characterise the association of insulin resistance (IR) in patients with PCOS in the southern Indian state of Andhra Pradesh.

\section{METHODS}

A cross sectional study was conducted among 50 patients diagnosed as PCOS, attending outpatient department of PES Institute of Medical Sciences and Research, Kuppam, Andhra Pradesh, India which is a tertiary care teaching hospital largely catering to the rural population of the state from January 2012 to March 2013 for a period of 15 months.

The inclusion criteria of this study were women between ages of 18 to 35 years; diagnosed for the first time as PCOS according to Rotterdam criteria of 2003, i.e., at least two of the following three features: oligomenorrhea or amenorrhea, clinical or biochemical hyperandrogenism and polycystic ovaries on ultrasound.

After taking an informed consent, detailed history was recorded for 50 participants. Physical examination included anthropometric data - height, weight and body mass index (BMI); clinical features suggestive of hirsutism and acanthosis nigricans were specifically noted.

Hirsutism was clinically graded using modified Ferriman Gallway (FG) system. A score of less than 8 or $\geq 8$ was diagnostic of Acanthosis nigricans, which is considered a cutaneous marker of IR; it is characterized by hyperpigmented velvety plaques seen in body folds and creases. The common sites of involvement include posterior aspect of neck and axilla. Acanthosis nigricans was graded based on standard scale of 0-4 as described by Burke et al. ${ }^{7}$ Acanthosis of neck-grade 0: not present, grade 1: present on close visual inspection, not visible to the casual observer, extent not measurable, grade 2: mild - limited to the base of the skull, does not extend to the lateral margin of the neck (usually, 3 inches in breadth), grade 3 : extending to the lateral margins, not visible from the front, and grade 4: extending anteriorly. Acanthosis of axilla - grade 0: absent, grade 1: present on close visual examination, grade 2 : localized to the central portion of axilla, grade 3: involving the entire axilla, and grade 4: extends beyond axilla.

Thereafter the participants underwent further investigations including fasting plasma glucose level and fasting plasma insulin level. HOMA (homeostasis model assessment) was applied and values calculated from fasting insulin and glucose concentrations using the following formula:
HOMA $-I R$

$=\frac{(\text { Fasting serum insulin }(\mu \mathrm{U} / \mathrm{ml}) \times \text { fasting plasma glucose }(\mathrm{mmol} / \text { liter })}{22.5}$

Values of $\geq 2.5$ were considered as significant insulin resistance (IR).

\section{RESULTS}

This study was conducted on 50 patients diagnosed for the first time with PCOS. Most of the participants in this study were between 25-29 years of age, which accounted for $42 \%$ of study population. Obese PCOS with BMI of $\geq 30 \mathrm{Kg} / \mathrm{m}^{2}$ accounted for only $(\mathrm{n}=4) 8 \%$ of this study group. $46 \%$ of participants $(n=23)$ belonged to normal and overweight category each. There was a strong family history of diabetes mellitus (DM) and PCOS in the firstdegree relatives in $42 \%$ and $8 \%$ participants respectively. The demographic details of the study participant are shown in Table 1.

Table 1: Demographic data.

\begin{tabular}{|c|c|c|}
\hline Demographic data & n & $\%$ \\
\hline \multicolumn{3}{|l|}{ Age (years) } \\
\hline$<20$ years & 7 & $14.0 \%$ \\
\hline 20-24 years & 18 & $36.0 \%$ \\
\hline $25-29$ years & 21 & $42.0 \%$ \\
\hline$\geq 30$ years & 4 & $8.0 \%$ \\
\hline \multicolumn{3}{|l|}{ BMI $\left(\mathrm{Kg} / \mathrm{m}^{2}\right)$} \\
\hline Underweight $(<18)$ & 0 & $0 \%$ \\
\hline Normal $(18-24)$ & 23 & $46.0 \%$ \\
\hline Over weight (25-29) & 23 & $46.0 \%$ \\
\hline Obese $(\geq 30)$ & 4 & $8.0 \%$ \\
\hline \multicolumn{3}{|c|}{ Family history of DM and PCOS } \\
\hline PCOS & 8 & $16.0 \%$ \\
\hline $\mathrm{DM}$ & 21 & $42.0 \%$ \\
\hline
\end{tabular}

Table 2: Presenting complaints.

\begin{tabular}{|l|l|l|}
\hline Presenting complaints & n & $\%$ \\
\hline Abnormal uterine bleeding & 31 & $62 \%$ \\
\hline Hirsutism & 3 & $6 \%$ \\
\hline Infertility & 13 & $26 \%$ \\
\hline Weight gain & 3 & $6 \%$ \\
\hline *Many patients had more than one complaint & e.g. & some \\
AUB patients also had hirsutism and weight gain.
\end{tabular}

The most common presenting complaint of the study patients $(n=31) 62 \%$ was abnormal uterine bleeding (AUB). The next common presentation was infertility at $(n=13) 26 \%$, the other co-morbidities that brought these patients to the hospital were hirsutism and weight gain accounting for $(\mathrm{n}=3) 6 \%$ each. Table 2 shows the distribution of this study population by the 'main' presenting complaint. However, many patients had more than one complaint e.g. some AUB patients also had associated hirsutism and weight gain. Significant hirsutism (FG score $\geq 8$ ) was seen in 12 patients, but 6 of 
these patients had presented with chief complaint of AUB, three with hirsutism, two with infertility and one for recent excessive weight gain. Prevalence of IR in PCOS in this study, as determined by HOMA-IR of $\geq 2.5$, was $(n=18) 36 \%$. Table 3 shows the distribution of glycaemic status and insulin levels among the study population. When we compared glycaemic status and insulin resistance among the study population $(n=41)$ $82 \%$ of study population were found to be normoglycaemic. All the cases who did not have IR (i.e. HOMA-IR <2.5) $(\mathrm{n}=32)$ had normal fasting glucose levels. All cases $(n=9)$ with impaired glycaemic status (i.e. fasting glucose $\geq 110 \mathrm{mg} \%$ ) were also detected to have IR.
Table 4 shows the association of IR with various clinical features of PCOS patients. Only $(n=4) 8 \%$ of this study participants were obese, $(n=23) 46 \%$ were overweight and the rest had normal BMI. IR was demonstrated in $75 \%$ of obese women, $39.1 \%$ of overweight women and $26.1 \%$ of normal weight PCOS women. IR was present in $(\mathrm{n}=12) 75 \%$ of this study participants with associated significant hirsutism (FG score $\geq 8$ ). Mild hirsutism (FG score $<8)$ was also seen in $(n=23) 74.8 \%$ of PCOS women without IR. Among the study population IR was more prevalent in individuals with severe grading of acanthosis, that is, grade 2 and grade 3 accounting for $77.8 \%$ and $84.6 \%$ respectively.

Table 3: Comparison of glycaemic status among PCOS with and without IR.

\begin{tabular}{|c|c|c|c|c|c|c|}
\hline & \multirow{3}{*}{ Levels } & \multicolumn{4}{|c|}{ HOMA } & \multirow{3}{*}{ Total } \\
\hline & & \multicolumn{2}{|c|}{$<2.5$} & \multicolumn{2}{|c|}{$\geq 2.5$} & \\
\hline & & $\mathbf{n}$ & $\%$ & $\mathrm{n}$ & $\%$ & \\
\hline \multirow{3}{*}{ Fasting glucose } & $<110$ & 32 & $78.0 \%$ & 9 & $22.0 \%$ & 41 \\
\hline & $110-125$ & 0 & $0.0 \%$ & 9 & $100.0 \%$ & 9 \\
\hline & $>125$ & 0 & $0.0 \%$ & 0 & $0.0 \%$ & 0 \\
\hline \multirow{2}{*}{ Fasting insulin } & $\leq 17$ & 31 & $81.6 \%$ & 7 & $18.4 \%$ & 38 \\
\hline & $>17$ & 1 & $8.3 \%$ & 11 & $91.7 \%$ & 12 \\
\hline
\end{tabular}

Table 4: Insulin resistance and clinical presentation.

\begin{tabular}{|c|c|c|c|c|c|c|c|c|}
\hline & \multirow{3}{*}{ Levels } & \multicolumn{4}{|c|}{ HOMA } & \multirow{3}{*}{ Total } & \multirow{3}{*}{$\chi^{2}$ value } & \multirow{3}{*}{ p value } \\
\hline & & \multicolumn{2}{|c|}{$<2.5$} & \multicolumn{2}{|c|}{$\geq 2.5$} & & & \\
\hline & & $\mathbf{n}$ & $\%$ & $\mathbf{n}$ & $\%$ & & & \\
\hline \multirow{3}{*}{ BMI } & Normal & 17 & $73.9 \%$ & 6 & $26.1 \%$ & 23 & \multirow{3}{*}{3.719} & \multirow{3}{*}{0.156} \\
\hline & Overweight & 14 & $60.9 \%$ & 9 & $39.1 \%$ & 23 & & \\
\hline & Obese & 1 & $25.0 \%$ & 3 & $75.0 \%$ & 4 & & \\
\hline \multirow{3}{*}{ Hirsutism } & Nil & 6 & $85.7 \%$ & 1 & $14.3 \%$ & 7 & \multirow{3}{*}{10.424} & \multirow{3}{*}{0.001} \\
\hline & $<8$ & 23 & $74.2 \%$ & 8 & $25.8 \%$ & 31 & & \\
\hline & $\geq 8$ & 3 & $25.0 \%$ & 9 & $75.0 \%$ & 12 & & \\
\hline \multirow{4}{*}{ Acanthosis } & 0 & 16 & $100.0 \%$ & 0 & $0.0 \%$ & 16 & \multirow{4}{*}{35.903} & \multirow{4}{*}{$<0.001$} \\
\hline & 1 & 12 & $100.0 \%$ & 0 & $0.0 \%$ & 12 & & \\
\hline & 2 & 2 & $22.2 \%$ & 7 & $77.8 \%$ & 9 & & \\
\hline & 3 & 2 & $15.4 \%$ & 11 & $84.6 \%$ & 13 & & \\
\hline
\end{tabular}

\section{DISCUSSION}

PCOS is a disorder of complex pathology as well as diverse clinical manifestations. IR seems to be a central feature in the etiology of PCOS. IR directly impacts the ovarian morphology as well as the ovarian function. This study attempted to document the association of IR in established PCOS cases, in the institution.

The prevalence of IR amongst PCOS patients in this study population was $36 \%$. Marshall et al reported that $65-70 \%$ of women with PCOS are affected by IR, and so they recommend treatment of IR with life style modification, and Metformin for all women with PCOS. ${ }^{5,8}$ The lower prevalence of IR in this study, may be explained due to the different criteria used for diagnosis of PCOS. Women diagnosed as PCOS using Rotterdam criteria generally have a milder metabolic dysfunction, and thus have lesser incidence of IR when compared to PCOS women diagnosed using National Institutes of Health (NIH) criteria as reported by Marshall et al. ${ }^{9}$ Another reason for this reduced IR rate could be the predominance of rural population in this study group. Many of these women were doing physical labour and working in the fields, some were students walking over long distances to school. However, this data of physical activity was not specifically captured in this study. 
PCOS women in general have a higher prevalence of obesity and IR is frequently seen in obese patients.IR affects some $70-80 \%$ of obese (BMI $\geq 30$ ) PCOS and some $20-25 \%$ of lean (BMI <25) PCOS women, as reported by Marshall et el, when using the NIH criteria. ${ }^{8}$ In this study, using Rotterdam criteria, only $(n=4) 8 \%$ of this study population were obese, and $(n=23) 46 \%$ were in the lean PCOS category. In this study, IR was demonstrated in $75 \%$ of this study obese PCOS women, in $39.1 \%$ of overweight patients and in $26.1 \%$ of lean PCOS women. Even though the number of obese PCOS in this study was small, there seems to be no difference in the prevalence of IR when NIH or Rotterdam criteria is used to diagnose PCOS. Probably the genetic component that alters the insulin action is responsible as part of the IR in the obese PCOS patients is independent of their obesity, and related specifically to their PCOS. Given the high prevalence of IR in obese PCOS women, efforts to counter IR with weight reduction by life style modification is important. Use of Metformin as insulin sensitizer should also be considered in obese PCOS women. $^{8}$

IR varies by different PCOS phenotypes. Studies stratifying affected women according to the Rotterdam diagnostic criteria have shown that women with the most marked metabolic abnormalities are those with hyperandrogenism. ${ }^{10}$ This study also found $75 \%$ PCOS patients with significant hirsutism (FG score $\geq 8$ ) had IR. In PCOS women with mild hirsutism (FG score $<8$ ), the IR prevalence was $25.8 \%$. Only $14.3 \%$ of PCOS women without hirsutism were detected to have IR. It appears from this data that the non-hyperandrogenic phenotype of PCOS is a milder phenotype, with lesser prevalence of IR. $^{10}$

The skin lesion, acanthosis nigricans, was reported to occur frequently in women with hyperandrogenism and diabetes mellitus by Kierland et al. ${ }^{11}$ Acanthosis nigricans is a cutaneous marker for IR and occurs frequently in obese hyperandrogenic women. Acanthosis nigricans grade 2 and 3 were associated with IR in $77.8 \%$ and $84.6 \%$. 9 out of 12 PCOS patients with significant hirsutism (FG score $\geq 8$ ) had grade 2 or 3 acanthosis nigricans. The three patients without acanthosis were of normal BMI. All PCOS women without acanthosis nigricans and grade 1 acanthosis nigricans did not have IR in this study.

In this study $(\mathrm{n}=9) \quad 18 \%$ of PCOS women were dysglycaemic, with fasting glucose $\geq 110 \mathrm{md} / \mathrm{dl}$. All these dysglycaemic women had demonstrable IR and they were overweight or obese. As per World Health Organization (WHO), patients with impaired fasting glucose (IFG) are classified as 'Prediabetes'. Prediabetes is a state of intermediate hyperglycaemia and in $70 \%$ cases these patient can progress to DM. National urban diabetes survey estimates the prevalence of prediabetes in India at $14 \%$. $^{12}$
The prevalence of impaired glucose tolerance (IGT), including IFG, and type 2 DM in PCOS women has been assessed in three large cross-sectional studies. ${ }^{13-15}$ The prevalence was $23-35 \%$ for IGT and $4-10 \%$ for DM in these studies. The prevalence of dysglycaemia was found to increase with increasing BMI. In this study prediabetic patients had BMI in the overweight or obese category. However, these findings are not seen consistently, and some studies do not show increased prevalence rates of IGT and DM in women with PCOS. ${ }^{16}$ The reasons for these discrepant findings, apart for racial/ethnic differences, are unclear, and need further large-scale population-based studies.

Life style modifications and Metformin have been demonstrated to be effective in normalizing several parameters in women with PCOS. Life style modifications like brisk walking, dieting to achieve weight loss and normal BMI are the cornerstones of PCOS management. Treatment with metformin for at least 8 weeks has been associated with reduction in weight, fasting glucose, and fasting insulin levels by $14 \%$, calculated insulin resistance (HOMA-IR) by $22 \%$ and reduced new onset diabetes by $40 \%$. Hirsutism in PCOS women has also shown improvement with metformin use for up to 6 months. ${ }^{17,18}$

\section{CONCLUSION}

In this study group of relatively young Indian women with PCOS, the prevalence of IR was $36 \%$, which is not very high considering the reported rates of $50 \%$ and above in literature. However, many of these PCOS women were already in the prediabetes category. A simple test for glycemic status and its correlation with clinical features of insulin resistance would be more cost effective in diagnosing and treating patients with insulin sensitizers for the metabolic syndrome in PCOS. The study concludes that routine investigations for insulin resistance were not mandatory. The importance of early treatment including healthy diet, regular physical activity, and medications, which address the associated manifestations and co-morbidities for all PCOS women is re-emphasised).

Funding: No funding sources

Conflict of interest: None declared

Ethical approval: The study was approved by the Institutional Ethics Committee

\section{REFERENCES}

1. Bharathi RV, Swetha S, Neerajaa J, Madhavica JV, Janani DM, Rekha SN, et al. An epidemiological survey: effect of predisposing factors for PCOS in Indian urban and rural population. Middle East Fertil Soc J. 2017;22:313-6.

2. Burghen GA, Givens JR, Kitabchi AE. Correlation of hyperandrogenism with hyperinsulinism in 
polycystic ovarian disease. J Clin Endocrinol Metab. 1980;50:113-6.

3. Kovacs G, Smith J. A guide to the polycystic ovary: Its effects on health and fertility. Castle Hill Barns, UK: TFM Publishing. 2002.

4. Carey AH, Chan KL, Short F, White DM, Williamson R, Franks S. Evidence for a single gene effect in polycystic ovaries and male pattern baldness. Clin Endocrinol (Oxf). 1993;38:653-8.

5. Dunaif A, Xia J, Book C, Schenker E, Tang Z. Excessive insulin receptor serine phosphorylation in cultured fibroblasts and in skeletal muscle: a potential mechanism for insulin resistance in the polycystic ovary syndrome. J Clin Invest. 1995;96:801-10.

6. Zhang L, Rodriquez H, Ohno S, Miller WL. Serine phosphorylation of human $\mathrm{P} 450 \mathrm{c} 17$ increases 17,20 lyase activity: implications for adrenarche and the polycystic ovary syndrome. Proc Natl Acad Sci USA. 1995;92:10619-23.

7. Burke JP, Hale DE, Hazuda HP, Stern MP. A quantitative scale of acanthosis nigricans. Diabetes Care. 1999;22:1655-9.

8. Marshall JC, Dunaif A. All women with PCOS should be treated for insulin resistance. Fertil Steril. 2012;97(1):18-22.

9. Jovanovic VP, Carmina E, Lobo RA. Not all women diagnosed with PCOS share the same cardiovascular risk profiles. Fertil Steril. 2010;94:826-32.

10. Moran L, Teede H. Metabolic features of the reproductive phenotypes of polycystic ovary syndrome. Hum Reprod Update. 2009;15:477-88.

11. Kierland RR, Lakatos I, Szijarto L. Acanthosis nigricans: an analysis of data in twenty-two cases and a study of its frequency in necropsy material. J Invest Dermatol. 1947;9:299-305.

12. Ramachandran A, Snehalatha C, Kapur A, Vijay V, Mohan V, Das AK, et al. High prevalence of diabetes and impaired glucose tolerance in India: National Urban Diabetes Survey. Diabetol. 2001;44:1094-101.

13. Legro RS, Kunselman AR, Dodson WC, Dunaif A. Prevalence and predictors of risk for type 2 diabetes mellitus and impaired glucose tolerance in polycystic ovary syndrome: a prospective, controlled study in 254 affected women. J Clin Endocrinol Metab. 1999;84:165-9.

14. Ehrmann DA, Barnes RB, Rosenfield RL, Cavaghan MK, Imperial J. Prevalence of impaired glucose tolerance and diabetes in women with polycystic ovary syndrome. Diabetes Care. 1999;22:141-6.

15. Ehrmann DA, Kasza K, Azziz R, Legro RS, Ghazzi MN. For the PCOS/Troglitazone study group. Effects of race and family history of type 2 diabetes on metabolic status of women with polycystic ovary syndrome. J Clin Endocrinol Metab. 2005;90:66-71.

16. Luque-Ramírez M, Alpanes M, Escobar-Morreale HF. The determinants of insulin sensitivity, B-cell function, and glucose tolerance are different in patients with polycystic ovary syndrome and in women who do not have hyperandrogenism. Fertil Steril. 2010;94:2214-21.

17. Norman RJ, Davies MJ, Lord J, Moran LJ. The life style modification in polycystic ovary syndrome. Trends Endocrinol Metab. 2002;13:251-7.

18. Hoeger KM. Exercise therapy in polycystic ovary syndrome. Semin Reprod Med. 2008;26:93-100.

Cite this article as: Anuradha V, Gangadharan $\mathrm{K}$, Saxena RK, Krishna L. Polycystic ovarian syndrome and insulin resistance: a South Indian study. Int J Reprod Contracept Obstet Gynecol 2020;9:1356-60. 